\title{
A new phase space method for recovering index of refraction from travel times
}

\author{
Eric Chung ${ }^{1}$, Jianliang Qian ${ }^{2}$, Gunther Uhlmann ${ }^{3}$ and Hongkai Zhao ${ }^{4}$ \\ ${ }^{1}$ Applied and Computational Mathematics, California Institute of Technology, Pasadena, \\ CA 91125, USA \\ 2 Department of Mathematics and Statistics, Wichita State University, Wichita, KS 67260, USA \\ ${ }^{3}$ Department of Mathematics, University of Washington, Seattle, WA 98195, USA \\ ${ }^{4}$ Department of Mathematics, University of California, Irvine, CA 92697-3875, USA \\ E-mail: tschung@acm.caltech.edu, qian@math.wichita.edu,gunther@math.washington.edu and \\ zhao@math.uci.edu
}

Received 17 October 2006, in final form 1 December 2006

Published 12 January 2007

Online at stacks.iop.org/IP/23/309

\begin{abstract}
We develop a new phase space method for reconstructing the index of refraction of a medium from travel time measurements. The method is based on the so-called Stefanov-Uhlmann identity which links two Riemannian metrics with their travel time information. We design a numerical algorithm to solve the resulting inverse problem. The new algorithm is a hybrid approach that combines both Lagrangian and Eulerian formulations. In particular the Lagrangian formulation in phase space can take into account multiple arrival times naturally, while the Eulerian formulation for the index of refraction allows us to compute the solution in physical space. Numerical examples including isotropic metrics and the Marmousi synthetic model are shown to validate the new method.
\end{abstract}

(Some figures in this article are in colour only in the electronic version)

\section{Introduction}

We address in this paper the inverse problem of determining the index of refraction of a medium from travel time information. The anisotropic index of refraction of the medium is modelled as a positive definite symmetric matrix, i.e. a Riemannian metric, in a bounded domain of Euclidean space. The problem of determining the Riemannian metric from first arrivals is known in differential geometry as the boundary rigidity problem. The travel time information is encoded in the boundary distance function, which measures the distance, with respect to the Riemannian metric, between boundary points. The problem of determining the index of refraction from multiple arrival times is called in differential geometry the lens rigidity problem. The information is encoded in the scattering relation which gives the exit 
point and direction of a geodesic if we know the incoming point and direction plus also the travel time.

Recent progress on the boundary rigidity problem and the lens rigidity problem, and the associated linearized problem, a problem in integral geometry, has motivated us to transfer those theoretical advances into developing numerical algorithms to determine the anisotropic index of refraction, i.e. the Riemannian metric, from travel time information.

The boundary rigidity problem consists in determining a compact Riemannian manifold with boundary up to an action of a diffeomorphism which is the identity at the boundary by knowing the geodesic distance function between boundary points (see [29, 30] and references therein). One needs an a priori hypothesis to do so since it is easy to find counterexamples if the index of refraction is too large in certain regions. An a priori condition that has been proposed is simplicity of the metric [20]. A manifold is simple if the boundary is strictly convex with respect to the Riemannian metric and there are no conjugate points along any geodesic. The linearized inverse problem of the boundary rigidity problem is the integral geometry problem of determining a symmetric tensor field from its integrals along geodesics for a symmetric tensor of order two [27]. By proving stability estimates for this problem, Stefanov and Uhlmann [29] have been able to establish a local generic uniqueness result and a conditional stability estimate of Hölder type for the nonlinear boundary rigidity problem for simple manifolds. However, when the simplicity condition is not fulfilled, Stefanov and Uhlmann [32] have proved an injectivity up to a potential field and stability for non-simple Riemannian manifolds under some topological assumptions and under the condition of existence of a subset of geodesics without conjugate points in the sense that their co-normal bundle covers the cotangent space of the manifold; see also Dairbekov [5] for results on integral geometry for non-trapping Riemannian manifolds. A similar result for the nonlinear problem has been proved recently (see [31]). So far it seems to be difficult to handle the case with conjugate points since they are related to the so-called caustics in geometrical optics. Therefore, we are interested in numerically recovering a Riemannian metric even when caustics are present. We remark that for simple manifolds knowing the scattering relation is the same as knowing the boundary distance function. It is only for non-simple manifolds that the scattering relation gives more information including multiple arrival times. We also expect that our work will shed light on theoretical investigations of the boundary rigidity problem, the lens rigidity problem and the inverse kinematic problem in the presence of caustics.

Our work is inspired by Stefanov and Uhlmann [28]. In that article, assuming that a threedimensional domain with a Riemannian metric is not trapping (i.e. every geodesic has finite length), they established an identity in phase space to link, roughly speaking, the difference of two Riemannian metrics with the difference of the scattering relation. They used this to prove that a Riemannian metric close to the isotropic metric can be recovered up to an action of isometry in a bounded smooth, strictly convex domain in $\mathbb{R}^{3}$ by knowing the length of the geodesics joining points on the boundary; see also Wang [35] for related stability results using the same general method. In terms of stability estimates for multidimensional inverse problems, see the review article by Isakov [11]; see also Isakov and Sun [13] for stability estimates by reducing hyperbolic inverse problems to integral geometry problems. For a general account of inverse problems for PDEs, see [12]. In this work we build on the identity derived in [28] to design a numerical inversion algorithm and show numerical examples to demonstrate the effectiveness of the new algorithm in reconstructing an isotropic index of refraction.

Although the Stefanov-Uhlmann identity is posed in phase space, they need to prove that a certain integral transform is invertible which they can only accomplish under some assumptions, including simplicity of the Riemannian manifold with respect to a given metric. 
In [28] the metric is recovered by using only the shortest path since, as was mentioned earlier, in this case the scattering relation is the same as the boundary distance function. However, the identity is valid for non-trapping manifolds, including anisotropic media and allowing for caustics, assuming that one knows the metric outside the domain. Thus, we decided to use the identity directly in phase space. As a consequence we have to find a way to pick the data that are in phase space which are not measurable directly. To do that we recall that in kinematic inverse problems, the data used frequently are travel time data, which means that travel times can be parametrized by source locations and ray parameters; in turn ray parameters can be derived from the eikonal equation and the travel time data as illustrated in $[10,14,19$, 33]. Therefore without any hesitation we use the identity as our foundation to carry out the inversion process.

The Stefanov-Uhlmann identity is also related to the so-called Liouville equation, but the current formulation is different from that used in [14]. In that work [14] Leung and Qian formulated the inverse problem for isotropic metrics in a Eulerian framework and used an adjoint state method to minimize a mismatching functional. The current new formulation is based on a novel identity to cross-correlate the information from two metrics so that the two metrics can pass information to each other at every moment, and the Jacobian matrix is the bridge.

The advantages of phase space formulation are multi-fold. As a first advantage multipathing can be taken into account systematically, as evidenced in [6, 14] and in numerical examples shown later. As demonstrated in $[9,16]$, multipathing is essential for high resolution seismic imaging. As a second advantage, our phase space formulation has the potential to recover generic (anisotropic) Riemannian metrics. These advantages distinguish our new method from other traditional methods in inverse kinematic problems [2, 3, 15, 24-26, 36] in that those traditional methods only recover isotropic metrics by using first-arrivals. Moreover, our numerical algorithm is based on a hybrid approach. A Lagrangian formulation (ray tracing) is used in phase space for the linearized Stefanov-Uhlmann identity. This allows us to deal with multipathing naturally. On the other hand, a Eulerian formulation is used for the index of refraction of the medium. As a consequence our computational domain is in physical space rather than in phase space. In this paper, we will focus on the numerical algorithm for isotropic metrics. For anisotropic cases the underlying Riemannian metric is unique up to an action of a diffeomorphism which is the identity at the boundary even with knowledge of scattering relations, e.g., the lens rigidity problem. This gives additional intriguing subtleties in developing numerical algorithms. The study will be reported in future work.

The paper is organized as follows. In section 2, we state the inverse problem, linearize the Stefanov-Uhlmann identity and propose a nonlinear functional as the foundation for solving the inverse problem in phase space. Section 3 is devoted to discretizing the nonlinear functional. In section 4, we show extensive examples to demonstrate the effectiveness of the new phase space method. Section 5 concludes the paper.

\section{Statement of the problem and linearization}

We are interested in reconstructing a Riemannian metric in a bounded domain by a set of boundary measurements.

\subsection{Basic setup}

Let $\Omega \subset \mathbb{R}^{n}$ be a bounded domain with smooth boundary $\Gamma=\partial \Omega$. Let $g(x)=\left(g_{i j}(x)\right)$ be a Riemannian metric in $\Omega$. Let $d_{g}(x, y)$ denote the geodesic distance between $x$ and $y$. 
The inverse problem is whether we can determine the Riemannian metric $g$ up to the natural obstruction of a diffeomorphism by knowing $d_{g}(x, y)$ for any $x \in \Gamma$ and $y \in \Gamma$. While theoretically there are a lot of works addressing this question (see [1, 4, 20-23, 27] and references therein), we explore the possibility of recovering the Riemannian metric numerically. We use phase space formulation so that multipathing in physical space is allowed. Rather than the boundary distance function we look at the scattering relation which measures the point and direction of exit of a geodesic plus the travel time if we know the point and direction of entrance of the geodesic. Such data are available in practice (see section 3.3). Below we formulate the problem more precisely.

Assume that we have two $C^{k}$ metrics $g_{1}$ and $g_{2}$ with $k \geqslant 2$ satisfying $g_{1}=g_{2}$ in $\mathbb{R}^{n}-\Omega$.

The Hamiltonian $H_{g}$ related to $g$ is

$$
H_{g}(x, \xi)=\frac{1}{2}\left(\sum_{i, j=1}^{n} g^{i j}(x) \xi_{i} \xi_{j}-1\right),
$$

where $g$ is either $g_{1}$ or $g_{2}$, and $g^{-1}=\left(g^{i j}\right)$.

Let $X:=(x, \xi)$. We denote by $X_{g_{j}}\left(s, X^{(0)}\right)=\left(x_{g_{j}}\left(s, X^{(0)}\right), \xi_{g_{j}}\left(s, X^{(0)}\right)\right), j=1,2$, the solution to the Hamiltonian system defined by $H_{g_{j}}(x, \xi)=0$,

$$
\frac{\mathrm{d} x}{\mathrm{~d} s}=\frac{\partial H_{g_{j}}}{\partial \xi}, \quad \frac{\mathrm{d} \xi}{\mathrm{d} s}=-\frac{\partial H_{g_{j}}}{\partial x},
$$

with the initial condition $X^{(0)}=\left(x^{0}, \xi^{0}\right)$, where $x^{0} \in \mathbb{R}^{n}$ and $\xi^{0} \in S_{g}^{n-1}\left(x^{0}\right)$. Here we define the unit sphere in the cotangent space $T_{x}^{*}\left(\mathbb{R}^{n}\right)$ :

$$
S_{g}^{n-1}(x):=\left\{\xi \in T_{x}^{*}\left(\mathbb{R}^{n}\right): H_{g}(x, \xi)=\frac{1}{2}\left(\Sigma_{i, j=1}^{n} g^{i j}(x) \xi_{i} \xi_{j}-1\right)=0\right\} .
$$

The continuous dependence on the initial data of the solution of the Hamiltonian system is characterized by the Jacobian,

$$
J_{g_{j}}(s)=J_{g_{j}}\left(s, X^{(0)}\right):=\frac{\partial X_{g_{j}}}{\partial X^{(0)}}\left(s, X^{(0)}\right) .
$$

It can be shown easily from the definition of $J$ and the corresponding Hamiltonian system (2.2) [7] that $J_{g_{j}}, j=1,2$, satisfies

$$
\frac{\mathrm{d} J}{\mathrm{~d} s}=M J, \quad J(0)=I,
$$

where, in terms of $H=H_{g_{j}}(j=1,2)$, the matrix $M$ is defined by

$$
M=\left(\begin{array}{cc}
H_{\xi, x}, & H_{\xi, \xi} \\
-H_{x, x}, & -H_{x, \xi}
\end{array}\right)
$$

Since $H_{g_{j}} \in C^{k}\left(T^{*}\left(\mathbb{R}^{n}\right)\right)(j=1,2)$, where $T^{*}\left(\mathbb{R}^{n}\right)$ denotes the cotangent bundle on $\Omega$, according to the standard theory $X_{g_{j}} \in C^{k-1}$ jointly in $s \in \mathbb{R}$ and $X^{(0)} \in\left\{\left(x^{0}, \xi^{0}\right): x^{0} \in \mathbb{R}^{n}\right.$, $\left.\xi^{0} \in S_{g}^{n-1}\left(x^{0}\right)\right\}([34], \mathrm{p} 30)$.

\subsection{Linearizing the Stefanov-Uhlmann identity}

Given boundary measurements for $g_{1}$, we are interested in recovering the metric $g_{1}$.

Take $X^{(0)}=\left(x^{0}, \xi^{0}\right)$, where $x^{0} \in \Gamma$ and $\xi^{0} \in S_{g}^{n-1}\left(x^{0}\right)$, such that the inflow condition holds,

$$
v\left(x^{0}\right) \cdot g^{-1} \xi^{0}<0,
$$

where $v\left(x^{0}\right)$ is the outer normal to $\Gamma=\partial \Omega$ at $x^{0}$, and $g$ is either $g_{1}$ or $g_{2}$. In general, we let $\mathcal{S}^{-}$and $\mathcal{S}^{+}$be subsets of $\mathbb{R}^{2 n}$ defined by

$$
\begin{aligned}
& \mathcal{S}^{-}=\left\{(x, \xi) \mid x \in \Gamma, \xi \in S_{g}^{n-1}(x), g^{-1} \xi \cdot v(x)<0\right\}, \\
& \mathcal{S}^{+}=\left\{(x, \xi) \mid x \in \Gamma, \xi \in S_{g}^{n-1}(x), g^{-1} \xi \cdot v(x)>0\right\}
\end{aligned}
$$


where $v(x)$ is the outward normal vector at $x \in \Gamma$; they correspond to the inflow and outflow conditions in phase space.

We link two metrics by introducing the function

$$
F(s):=X_{g_{2}}\left(t-s, X_{g_{1}}\left(s, X^{(0)}\right)\right),
$$

where $t=\max \left(t_{g_{1}}, t_{g_{2}}\right)$ and $t_{g}=t_{g}\left(X^{(0)}\right)$ is the length of the geodesic issued from $X^{(0)}$ with the endpoint on $\Gamma$. Consequently we have

$$
\int_{0}^{t} F^{\prime}(s) \mathrm{d} s=X_{g_{1}}\left(t, X^{(0)}\right)-X_{g_{2}}\left(t, X^{(0)}\right) .
$$

The time integral on the left-hand side is equal to the following [28],

$\int_{0}^{t} F^{\prime}(s) \mathrm{d} s=\int_{0}^{t} \frac{\partial X_{g_{2}}}{\partial X^{(0)}}\left(t-s, X_{g_{1}}\left(s, X^{(0)}\right)\right) \times\left(V_{g_{1}}-V_{g_{2}}\right)\left(X_{g_{1}}\left(s, X^{(0)}\right)\right) \mathrm{d} s$,

where

$$
V_{g_{j}}:=\left(\frac{\partial H_{g_{j}}}{\partial \xi},-\frac{\partial H_{g_{j}}}{\partial x}\right)=\left(g^{-1} \xi,-\frac{1}{2} \nabla_{x}\left(g^{-1} \xi\right) \cdot \xi\right) .
$$

This is the so-called Stefanov-Uhlmann identity.

We linearize the above identity about $g_{2}$ by following a full underlying path in $g_{2}$,

$\int_{0}^{t} F^{\prime}(s) \mathrm{d} s \approx \int_{0}^{t} \frac{\partial X_{g_{2}}}{\partial X^{(0)}}\left(t-s, X_{g_{2}}\left(s, X^{(0)}\right)\right) \times \partial_{g_{2}} V_{g_{2}}\left(g_{1}-g_{2}\right)\left(X_{g_{2}}\left(s, X^{(0)}\right)\right) \mathrm{d} s$,

where $\partial_{g} V_{g}(\lambda)$ is the derivative of $V_{g}$ with respect to $g$ at $\lambda$.

Using (2.8), we have the following formula,

$$
\begin{aligned}
X_{g_{1}}\left(t, X^{(0)}\right)- & X_{g_{2}}\left(t, X^{(0)}\right) \approx \int_{0}^{t} \frac{\partial X_{g_{2}}}{\partial X^{(0)}}\left(t-s, X_{g_{2}}\left(s, X^{(0)}\right)\right) \\
& \times \partial_{g_{2}} V_{g_{2}}\left(g_{1}-g_{2}\right)\left(X_{g_{2}}\left(s, X^{(0)}\right)\right) \mathrm{d} s,
\end{aligned}
$$

or

$$
\begin{aligned}
X_{g_{1}}\left(t, X^{(0)}\right)- & X_{g_{2}}\left(t, X^{(0)}\right)=\int_{0}^{t} J_{g_{2}}\left(t-s, X_{g_{2}}\left(s, X^{(0)}\right)\right) \\
& \times \partial_{g_{2}} V_{g_{2}}\left(g_{1}-g_{2}\right)\left(X_{g_{2}}\left(s, X^{(0)}\right)\right) \mathrm{d} s,
\end{aligned}
$$

which is the foundation for our numerical procedure.

By the group property of Hamiltonian flows the Jacobian matrix is equal to

$$
\frac{\partial X_{g_{2}}}{\partial X^{(0)}}\left(t-s, X_{g_{2}}\left(s, X^{(0)}\right)\right)=\frac{\partial X_{g_{2}}}{\partial X^{(0)}}\left(t, X^{(0)}\right) \times\left(\frac{\partial X_{g_{2}}}{\partial X^{(0)}}\left(s, X^{(0)}\right)\right)^{-1},
$$

or

$$
J_{g_{2}}\left(t-s, X_{g_{2}}\left(s, X^{(0)}\right)\right)=J_{g_{2}}\left(t, X^{(0)}\right) \times J_{g_{2}}\left(s, X^{(0)}\right)^{-1} .
$$

In the case of the isotropic metric, we have

$$
\left(g_{k}\right)_{i j}=\frac{1}{c_{k}^{2}} \delta_{i j}
$$

Then

$$
V_{g_{k}}=\left(c_{k}^{2} \xi,-\left(\nabla c_{k}\right) c_{k}|\xi|^{2}\right)
$$

Hence the derivative of $V$ with respect to $g, \partial_{g} V_{g}(\lambda)$ in equation (2.8) is given by

$$
\partial_{g} V_{g}(\lambda)=\left(2 c \lambda \xi,-(\nabla c \cdot \lambda+\nabla \lambda \cdot c)|\xi|^{2}\right)
$$

since the phase space variable $\xi$ is fixed along the given path $X_{g_{2}}\left(s, X^{(0)}\right)$. A more involved formula can be derived for anisotropic media in the same way.

We note that one may also derive formula (2.12) by using the so-called ray perturbation theory (see [8], for example). 


\subsection{Continuous Tikhonov regularization}

Given boundary measurements $X_{g_{1}}\left(t, X^{(0)}\right) \in \mathcal{S}^{+}$, where $X^{(0)} \in \mathcal{S}^{-}$, we recover $g_{1}$ by Newton's method via the linearization (2.13). During each iteration, we solve the first-kind integral equation (2.13) by a regularization method.

For a given metric $g_{2}$ we define a linear operator, the $X$-ray transform via the Hamiltonian flow $X_{g_{2}}$, to map a function $\tilde{g} \in C_{0}^{k}\left(\mathbb{R}^{n}\right)$ into the set of its line integrals:

$$
\begin{aligned}
& K: \tilde{g} \in C_{0}^{k}\left(\mathbb{R}^{n}\right) \rightarrow K \tilde{g} \in C^{k}\left(\mathcal{S}^{-}\right), \\
& K \tilde{g}=\int_{0}^{t_{g_{2}}(\cdot)} J_{g_{2}}\left(t_{g_{2}}(\cdot)-s, X_{g_{2}}(s, \cdot)\right) \times \partial_{g_{2}} V_{g_{2}}(\tilde{g})\left(X_{g_{2}}(s, \cdot)\right) \mathrm{d} s, \\
& K \tilde{g}\left(X^{(0)}\right)=\int_{0}^{t_{g_{2}}\left(X^{(0)}\right)} J_{g_{2}}\left(t_{g_{2}}\left(X^{(0)}\right)-s, X_{g_{2}}\left(s, X^{(0)}\right)\right) \times \partial_{g_{2}} V_{g_{2}}(\tilde{g})\left(X_{g_{2}}\left(s, X^{(0)}\right)\right) \mathrm{d} s, \\
& \quad \text { for } \quad X^{(0)} \in \mathcal{S}^{-} .
\end{aligned}
$$

We define the data element as

$$
\tilde{d}=X_{g_{1}}(t, \cdot)-X_{g_{2}}(t, \cdot),
$$

where $\tilde{d} \in C^{k}\left(\mathcal{S}^{-}\right)$. Hence we have a first-kind operator equation by equation (2.13),

$$
K \tilde{g}=\tilde{d} .
$$

By the method of regularization we introduce a nonzero parameter $\beta$ and a functional,

$$
G_{\beta}(\tilde{g})=\frac{1}{2}\|K \tilde{g}-\tilde{d}\|_{\mathcal{L}^{2}\left(\mathcal{S}^{-}\right)}^{2}+\frac{1}{2} \beta\|\nabla \tilde{g}\|_{\mathcal{L}^{2}(\Omega)}^{2},
$$

which is defined on the domain of the gradient operator $\nabla$. Then we seek an element $\tilde{g}_{\beta}$ solving the minimizing problem,

$$
G_{\beta}\left(\tilde{g}_{\beta}\right)=\inf _{\tilde{g} \in D(\nabla)} G_{\beta}(\tilde{g}),
$$

where $D(\nabla)$ is the domain of $\nabla$. Under appropriate conditions we may show that the $\tilde{g}_{\beta}$ converges to a least-squares solution $\tilde{g}_{0}$ of equation $(2.21)[17,18]$.

\subsection{A continuation method}

In general Newton's method for the nonlinear equation (2.9) based on solving the linearized problem (2.12) iteratively may not converge for an arbitrary initial guess. However, since stability and uniqueness are proved for a metric close to the isotropic metric [28, 35], this enables us to develop a continuation (homotopy) method. A series of intermediate metrics is created continuously from an appropriately known metric, such as an isotropic metric, to the unknown metric by interpolation of the data.

Let $g_{0}$ be an appropriately chosen isotropic metric, and let $\tilde{t}_{j}$ and $\tilde{X}_{j}=X_{g_{0}}\left(\tilde{t}_{j}, X_{j}^{(0)}\right)$ be the data in the given medium corresponding to those measurements with initial states $X_{j}^{(0)}$ for $j=1,2, \ldots, m$. That is, $\tilde{t}_{j}$ is the travel time such that $\tilde{X}_{j}=X_{g_{0}}\left(\tilde{t}_{j}, X_{j}^{(0)}\right) \in \mathcal{S}^{+}$by solving the forward problem in the given metric. Now we take a continuation from the given metric to the unknown metric by introducing an intermediate metric, $g_{\alpha}$, corresponding to the linear interpolation of the measurements parametrized by $\alpha \in[0,1]$,

$$
t_{j}^{\alpha}=\alpha t_{j}+(1-\alpha) \tilde{t}_{j}, \quad X_{j}^{\alpha}=\alpha X_{\hat{g}}\left(t_{j}, X_{j}^{(0)}\right)+(1-\alpha) \tilde{X}_{j} .
$$

Numerically this procedure introduces an inner loop. First we choose an increment $\mathrm{d} \alpha=1 / N$. 
Start with $\alpha_{1}=\mathrm{d} \alpha$ and use the interpolated data, $t_{j}^{\alpha_{1}}$ and $X_{j}^{\alpha_{1}}$, to find the first intermediate metric $g_{\alpha_{1}}$. This process is continued as follows: after we get $g_{\alpha_{k}}$, we use it as an initial guess to compute $g_{\alpha_{k+1}}$.

In addition a restarting step could be helpful. The restarting step is to use the metric computed from previous computations as a new initial state to start a new continuation process. For example, if the previous stage becomes divergent at $\alpha_{n}, 1 \leqslant n<N$, then $g_{\alpha_{n}}$ and the corresponding measurements, $t_{j}^{\alpha_{n}}$ and $X_{j}^{\alpha_{n}}$, are used as the new initial metric and measurements for the next continuation process. In general we still may not be able to complete the whole continuation, but we can obtain a certain approximation of the unknown metric.

\section{Numerical methods}

\subsection{Discretization}

We use a hybrid approach to solve this problem numerically. The metric $g$ is defined on an Eulerian grid. The linearized integral equation (2.12) is treated in phase space using a Lagrangian formulation. Hence multipathing can be dealt with easily. The main task in the numerical computation is the discretization of the linearized integral equation (2.12). Since the integral is defined along the ray in a given metric, we first solve the ray equation (2.2) in phase space starting from a particular $X_{j}^{(0)}$. At the same time we compute the Jacobian matrix along the ray according to (2.5) and (2.15). Standard ODE solvers are used to solve these ODEs. On the ray, values of $g$ are computed by interpolation from the neighbouring grid point values. Hence each integral equation along a particular ray yields a linear equation for grid values of $g$ near the ray path in the physical domain. We form a whole system of linear equations using all rays corresponding to the given measurements. This system may be under or over determined and may not have a unique solution. We regularize the system as in the continuous case discussed in section 2.3. A few remarks are in order on our numerical algorithm:

(i) Although our formulation is in phase space, the phase variables are auxiliary dependent variables. Physical space is where we solve the problem.

(ii) The main cost is in solving the ray equations corresponding to the measurements and forming the linear system.

We assume that the metric $\hat{g}$ to be reconstructed is known on the boundary $\Gamma$. Let $X_{i}^{(0)} \in \mathcal{S}^{-}$, for $i=1,2, \ldots, m$, be a set of given points which are the initial conditions producing the measurements. Here $m$ is the number of data points in phase space. In principle, the measurements, $X_{\hat{g}}\left(t_{i}, X_{i}^{(0)}\right)$, are obtained by performing external measurements. To test our numerical algorithm, which will be discussed in section 4 , we will generate the measurements by solving the forward problem (2.2) with the initial data $X_{i}^{(0)} \in \mathcal{S}^{-}$until a time $t_{i}$ such that $X_{\hat{g}}\left(t_{i}, X_{i}^{(0)}\right) \in \mathcal{S}^{+}$.

Let $\tau$ be a rectangular grid on $\Omega$ with mesh size $h>0$. We denote by $Q^{1}(\tau)$ the space of bilinear functions on $\tau$. We define $W=\left\{g|g|_{\tau} \in Q^{1}(\tau),\left.g\right|_{\Gamma}=\left.\hat{g}\right|_{\Gamma}\right\}$ and $W_{0}=\left\{g|g|_{\tau} \in Q^{1}(\tau),\left.g\right|_{\Gamma}=0\right\}$. The following is the numerical scheme.

Suppose the initial guess $g^{0} \in W$ is given. If $g^{n} \in W$ is known, then we find $g^{n+1} \in W$, where $g^{n+1}$ solves the following minimization problem,

$$
\min _{g \in W} B(g)=\frac{1}{2} \sum_{i=1}^{m}\left\|F_{i}\left(g^{n}\right) g-r_{i}\left(g^{n}\right)\right\|^{2}+\frac{\beta}{2}\|\nabla g\|^{2} .
$$


Here, for $1 \leqslant i \leqslant m$,

$$
F_{i}\left(g^{n}\right) g=\int_{0}^{t_{i}} \frac{\partial X_{g^{n}}}{\partial X^{(0)}}\left(t_{i}-s, X_{g^{n}}\left(s, X_{i}^{(0)}\right)\right) \times \partial_{g^{n}} V(g)\left(X_{g^{n}}\left(s, X_{i}^{(0)}\right)\right) \mathrm{d} s
$$

and

$$
r_{i}\left(g^{n}\right)=X_{\hat{g}}\left(t_{i}, X_{i}^{(0)}\right)-X_{g^{n}}\left(t_{i}, X_{i}^{(0)}\right)+F_{i}\left(g^{n}\right) g^{n} .
$$

In (3.1), $\beta>0$ is a regularization parameter. The minimizer $g$ of (3.1) satisfies the following variational problem,

$$
\sum_{i=1}^{m}\left\langle F_{i}\left(g^{n}\right) g, F_{i}\left(g^{n}\right) \phi\right\rangle+\beta\langle\nabla g, \nabla \phi\rangle=\sum_{i=1}^{m}\left\langle r_{i}\left(g^{n}\right), F_{i}\left(g^{n}\right) \phi\right\rangle, \quad \forall \phi \in W .
$$

The existence and uniqueness of a solution for (3.4) can be proved by standard techniques. Note that (3.4) is a linear system and can be solved directly.

Now, we consider the right-hand side of (3.2). We discretize the time interval $(0, t)$ into smaller subintervals. On the $j$ th subinterval, we have

$$
\int_{j \Delta t}^{(j+1) \Delta t} \frac{\partial X_{g^{n}}}{\partial X^{(0)}}\left(t-s, X_{g^{n}}\left(s, X^{(0)}\right)\right) \times \partial_{g^{n}} V(g)\left(X_{g^{n}}\left(s, X^{(0)}\right)\right) \mathrm{d} s .
$$

Using a trapezoidal rule, for instance, we have

$$
\begin{aligned}
\frac{\Delta t}{2}\left\{\frac{\partial X_{g^{n}}}{\partial X^{(0)}}(t-\right. & \left.t_{j}, X_{g^{n}}\left(t_{j}, X^{(0)}\right)\right) \times \partial_{g^{n}} V(g)\left(X_{g^{n}}\left(t_{j}, X^{(0)}\right)\right) \\
& \left.+\frac{\partial X_{g^{n}}}{\partial X^{(0)}}\left(t-t_{j+1}, X_{g^{n}}\left(t_{j+1}, X^{(0)}\right)\right) \times \partial_{g^{n}} V(g)\left(X_{g^{n}}\left(t_{j+1}, X^{(0)}\right)\right)\right\}
\end{aligned}
$$

where $t_{j}=j \Delta t$.

In addition, we need to calculate the Jacobian matrix along the ray

$$
\frac{\partial X_{g^{n}}}{\partial X^{(0)}}\left(t-t_{j}, X_{g^{n}}\left(t_{j}, X^{(0)}\right)\right)=\frac{\partial X_{g^{n}}}{\partial X^{(0)}}\left(t, X^{(0)}\right) \times\left(\frac{\partial X_{g^{n}}}{\partial X^{(0)}}\left(t_{j}, X^{(0)}\right)\right)^{-1},
$$

which, in turn, can be computed by solving equation (2.5) with the matrix $M$ defined by (2.6). In the case of an isotropic medium with the metric defined as in (2.16),

$$
M=\left(\begin{array}{cc}
2 c^{n} \xi\left(\nabla c^{n}\right)^{T}, & \left(c^{n}\right)^{2} I \\
-\left(\nabla^{2} c^{n}\right) c^{n}|\xi|^{2}-\left(\nabla c^{n}\right)\left(\nabla c^{n}\right)^{T}|\xi|^{2}, & -2 c^{n}\left(\nabla c^{n}\right) \xi^{T}
\end{array}\right) .
$$

\subsection{Computational cost}

We estimate the computational cost of the numerical method. For each iteration, we have to solve the ray equation (that is, the ODE) for each initial datum (that is, each initial ray direction and source location). Suppose we use $N_{T}$ time steps for the ODE solver. Then the computational cost for solving the ray equation for each iteration is $O\left(m N_{T}\right)$, where $m$ is the number of measurements.

We will store the numerical solution at each time step when solving the ray equation by an ODE solver. The advantage of this is that we do not need to compute the value $X_{g^{n}}$ again when we need to evaluate the integral (3.5)-(3.6) and find the Jacobian matrix (2.5).

Regarding the linear system, we use a direct solver. So, for each integration, the cost of computation is $O\left(N^{3}\right)$. Note that the linear system is dense. However, the use of a direct 
solver is not compulsory. One can apply other algebraic solvers for better efficiency. We will not pursue this issue any further in the current paper.

\subsection{Data collection}

In isotropic media travel times and ray parameters can be picked from so-called common-shot (source) and common-geophone gathers, where the ray parameter is defined as the change in travel time as the position of the shot, the receiver, or both, varies [33]. There are several possible approaches, such as the method of controlled directional reception [33] and the method of double slant stacking [10]. In turn, the ray parameter can be used for determining take-off angles at the shot position and the arrival angles at the geophone position [33]. See [19] for another related method. Therefore, it is possible to apply our mathematical formulation to practice.

\section{Numerical examples}

In this section, we present a series of experiments to test the new numerical method.

The numerical solution is uniquely determined by its value at each interior node. The measurement is generated by solving the forward problem with the exact metric. The initial data are chosen in the following way. Note that each initial datum point $X^{(0)}$ contains two components $x^{(0)}$ and $\xi^{(0)}$ which are the initial physical location and the initial phase state; in turn, the initial phase state determines the ray direction.

We assume that the domain $\Omega=[0,1]^{2}$. For each of the examples below, except otherwise noted, we will use a $11 \times 11$ rectangular grid, which contains $11^{2}$ nodes including those on the boundary. We will use 9 initial physical locations $x^{(0)}$ on each edge of the domain $\Omega$, which are the interior nodal points on that edge; therefore we have $9 \times 4$ initial physical locations in total, and they are the nodes on the boundary except those at the corners of $\Omega$. At each initial physical location, we use ten initial directions. These ten directions are specified by unit vectors pointing inwards and they are chosen to be uniformly distributed among all possible inward unit vectors. Hence, the total number of measurements is 360 . For given initial data, the forward problem is solved and the measurement is obtained when the bi-characteristic reaches the boundary with an outward pointing direction.

We will show numerical examples for isotropic metrics and the so-called Marmousi synthetic model.

\subsection{Isotropic metrics}

4.1.1. Example 1: a constant model. In this example, we assume that the exact speed is a constant function which is equal to 10 . The regularization parameter $\beta$ is $10^{-12}$. We consider two different initial guesses: the first one is a constant function which is equal to 8 and the second one is also a constant function which is equal to 5 .

For the case with the constant 8 as the initial guess, the algorithm stops after four iterations and recovers the solution exactly. The value of the successive error at the fourth iteration is $3.2742 \times 10^{-6}$.

For the case with the constant 5 as the initial guess, the algorithm stops after five iterations and recovers the solution exactly. The value of the successive error at the fifth iteration is $2.0438 \times 10^{-4}$.

In figure 1, we show the behaviour of the successive error against iterations. We see that our numerical algorithm converges very fast even for the initial guess far away from the exact solution. 

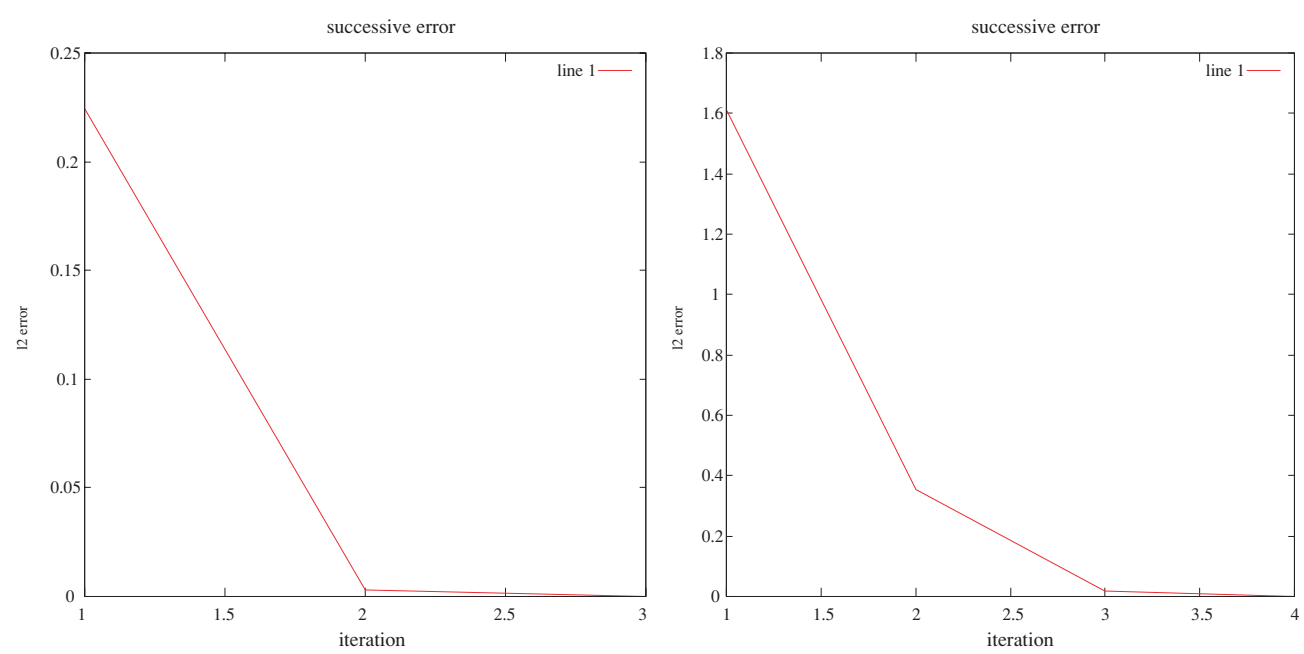

Figure 1. Successive error versus iteration for example 1. Left: the initial guess is 8 . Right: the initial guess is 5 .
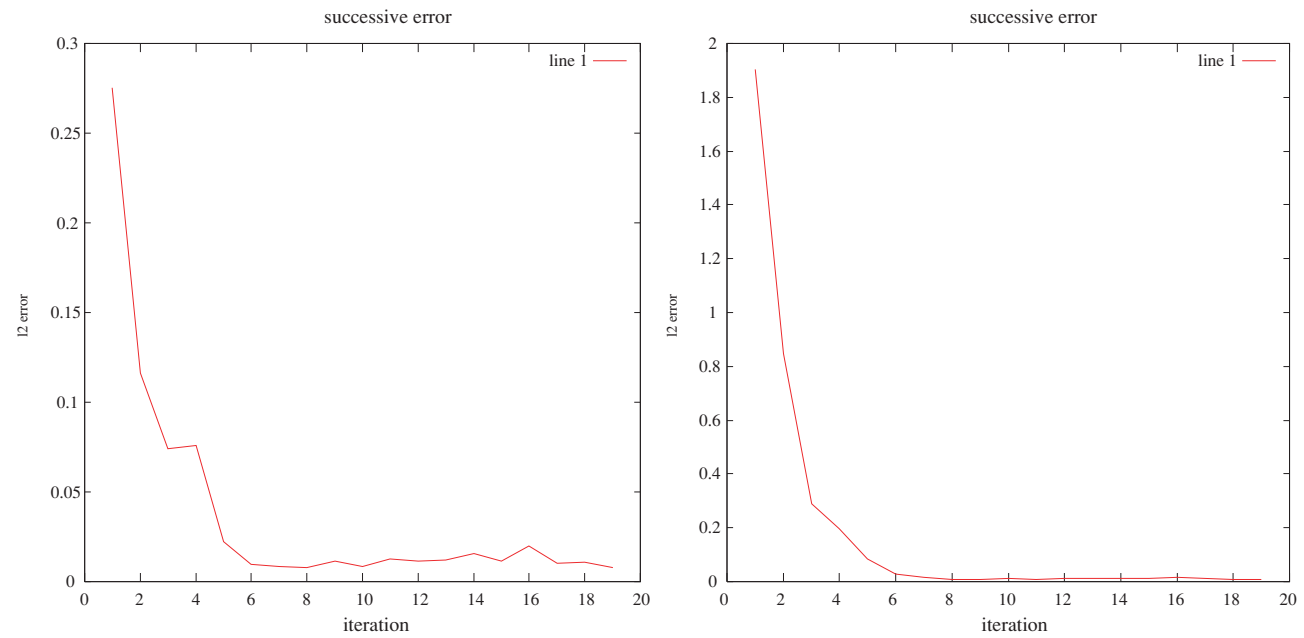

Figure 2. Value of successive error versus iteration for example 3. Left: using 8 as the initial guess. Right: using 5 as the initial guess.

4.1.2. Example 2: an additive sinusoidal model. We present a numerical experiment with the exact speed given by

$$
c(x, y)=10+\sin (3 \pi x)+\sin (3 \pi y) .
$$

The regularization parameter is $\beta=10^{-6}$. As in the previous two examples, we consider two different cases with the initial guess being a constant function which is equal to 8 and 5 , respectively. In figure 2 , the values of the successive error against iteration for both cases up to the first 20 iterations are shown. From the figure, we see that the algorithm converges after a few iterations. 

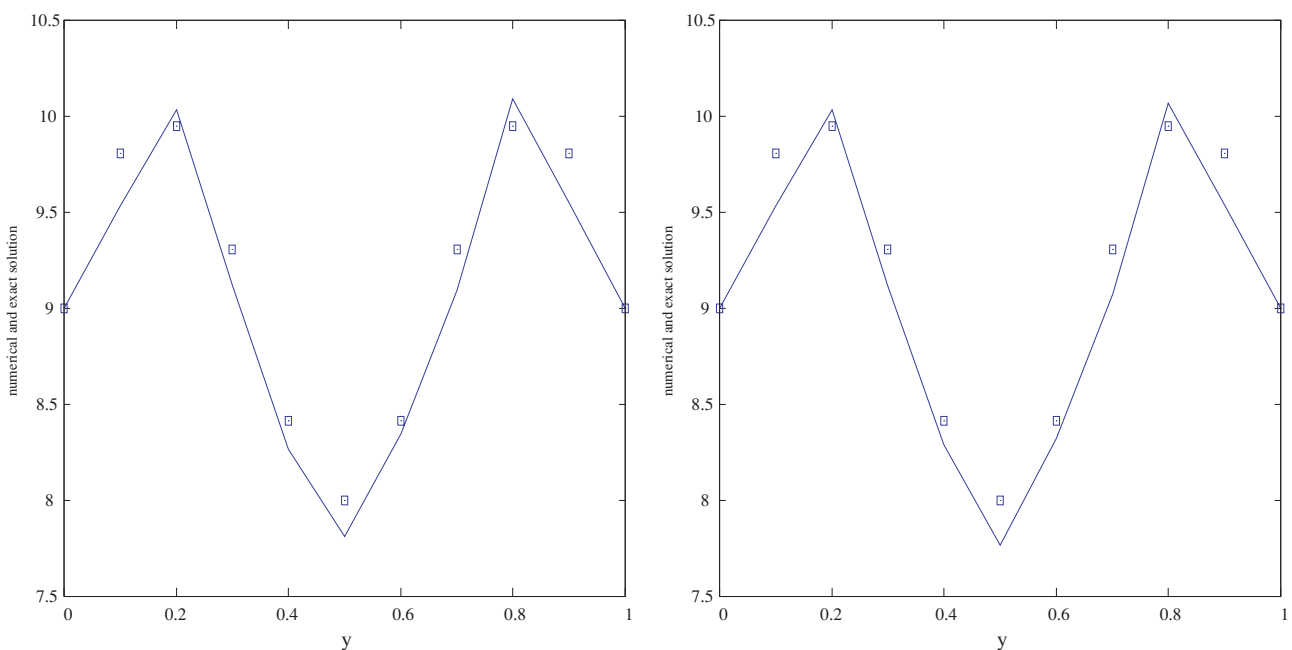

Figure 3. Numerical and exact solutions at $x=0.5$ for example 3 . Left: using 8 as the initial guess. Right: using 5 as the initial guess. The solid line represents the numerical solution and the squares represent the values of the exact solution at the nodal points.
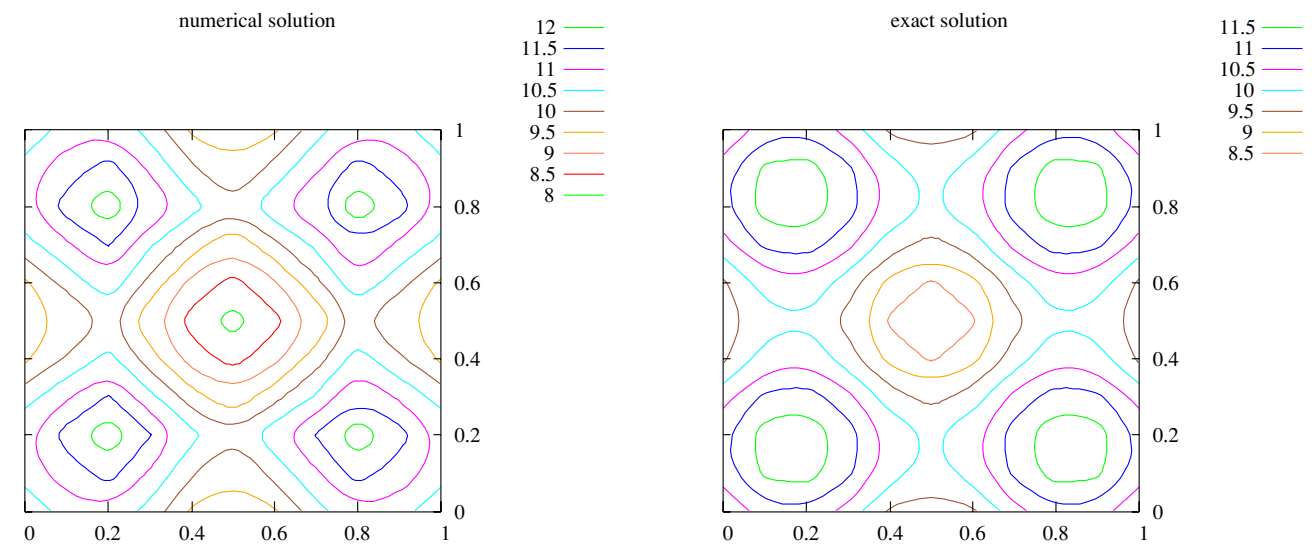

Figure 4. Contour plots for numerical solution using 5 as the initial guess and the exact solution for example 3. Left: numerical. Right: exact.

In figure 3 , the numerical and exact solutions at $x=0.5$ are shown. The case with 8 as the initial guess is shown on the left of figure 3 while the case with 5 as the initial guess is shown on the right of figure 3 . From the figures, we see that our numerical algorithm recovers the speed quite well. However, the numerical solution is less accurate in the middle of the domain. The $L^{2}$-norms of the difference between the numerical solution and the exact solution on the whole domain for the case with 8 as the initial guess and the case with 5 as the initial guess are 0.016182 and 0.016437 , respectively.

In figure 4, the contour plots of the numerical solution with the initial guess 5 and the exact solution are presented.

In figure 5, we present the difference between the exact solution and the numerical solution with the initial guess 5 for the first, second, third and 20th iteration. From these four figures, 

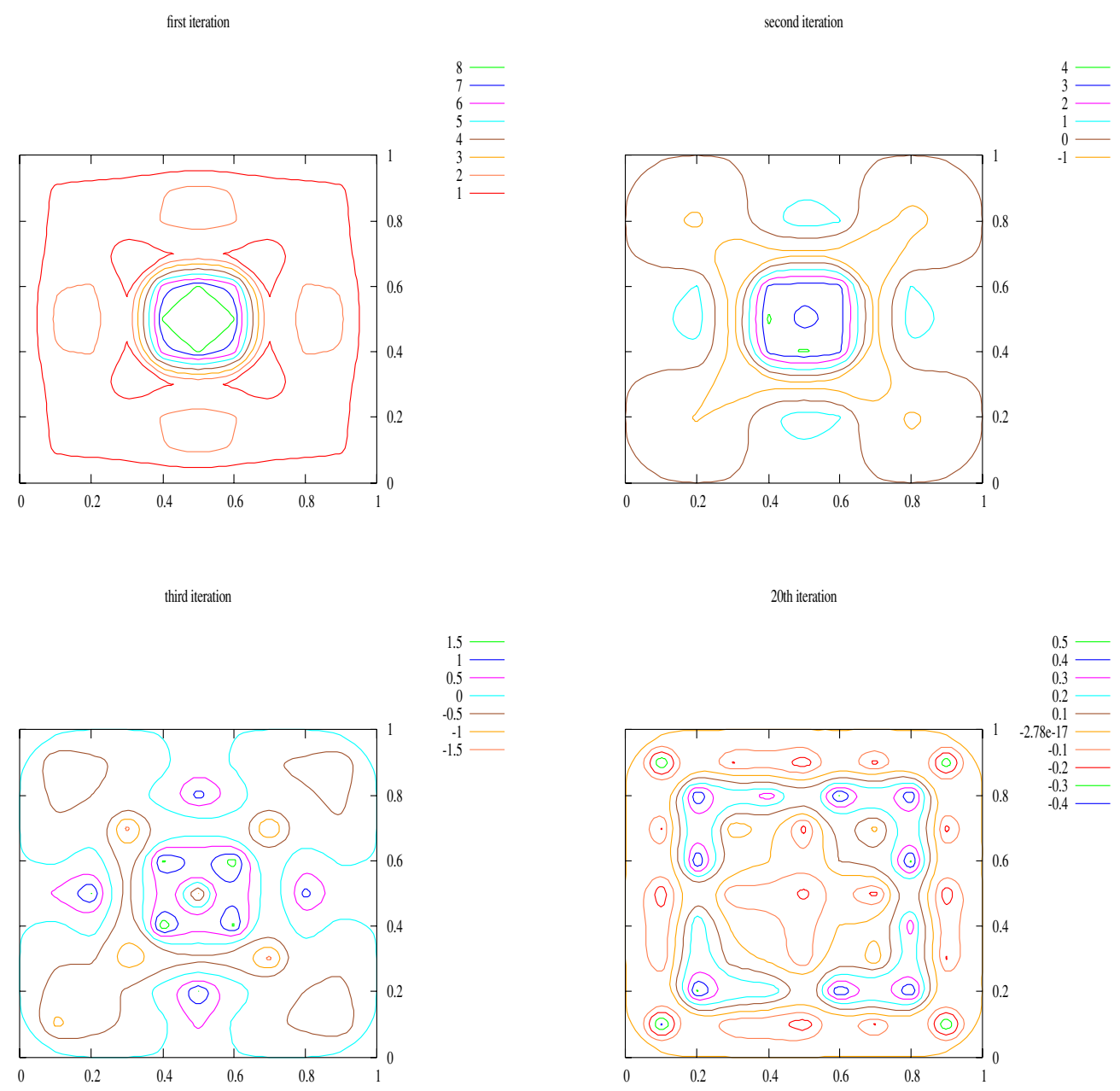

Figure 5. Contour plots for the difference between numerical using 5 as the initial guess and the exact solution for example 3. Top-left: first iteration. Top-right: second iteration. Bottom-left: third iteration. Bottom-right: 20th iteration.

we see that the error is comparatively smaller in regions closer to the boundary, and the error is comparatively larger in regions farther away from the boundary.

Now, we add 5\% uniformly distributed random noise to the measured travel time. In figure 6 , the numerical and exact solutions at $x=0.5$ are shown. The case with 8 as the initial guess is shown in the left of figure 6 , while the case with 5 as the initial guess is shown in the right of figure 6 . The $L^{2}$-norms of the difference between the numerical solutions and the exact solution on the whole domain are 0.14817 and 0.15296 for the two cases, respectively.

In figure 7, the value of the successive error against iteration for the first 10 iterations is shown. We see that the algorithm converges after a few iterations.

In figure 8 , we present the contour plots of the numerical solutions. The case with 8 as the initial guess is shown in the left of figure 8 , while the case with 5 as the initial guess is shown in the right of figure 8 .

In figure 9 , we show the three rays crossing at a certain point for this model. The initial physical location is $(0.5,0)$. The three initial directions are $(\cos (\theta), \sin (\theta))$ with 

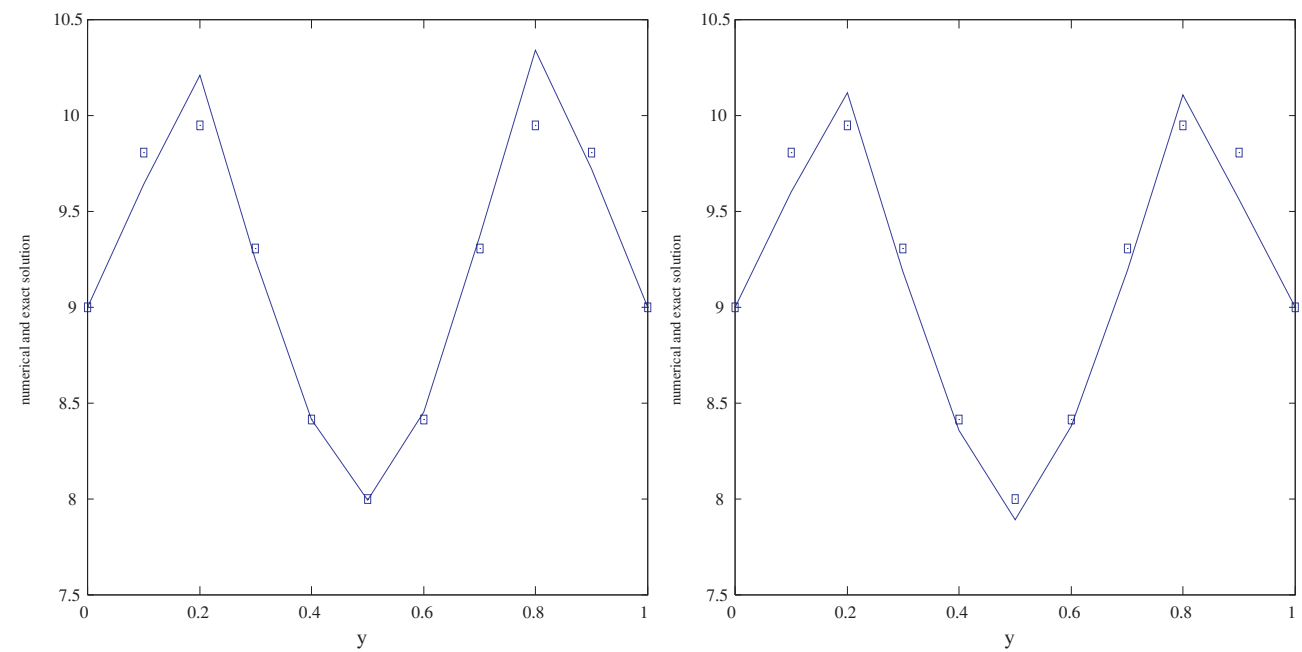

Figure 6. Numerical and exact solutions at $x=0.5$ for example 3 with $5 \%$ noise. Left: using 8 as the initial guess. Right: using 5 as the initial guess. The solid line represents the numerical solution and the square represents the values of the exact solution at the nodal points.
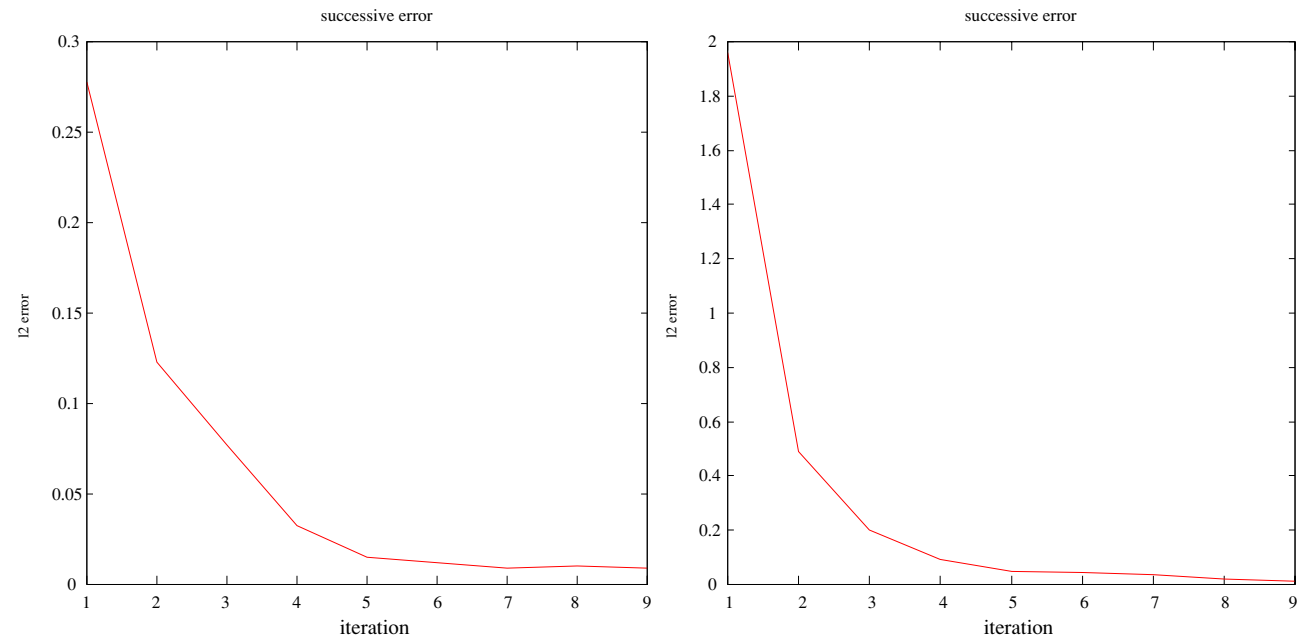

Figure 7. Value of successive error versus iteration for example 3 with 5\% noise. Left: using 8 as the initial guess. Right: using 5 as the initial guess.

$\theta=\pi / 2-0.05, \pi / 2$ and $\pi / 2+0.1$. This demonstrates that the proposed method can take into account multiple paths with ease, implying that caustics are allowed.

4.1.3. Example 3: a multiplicative sinusoidal model. We present a numerical experiment with the exact speed given by

$$
c(x, y)=1+0.2 \sin (3 \pi x) \sin (\pi y) .
$$

The regularization parameter is $\beta=10^{-10}$. We will use a $21 \times 21$ grid and the initial condition is the constant function 1 . At each grid point on the boundary, we use 10 directions. 

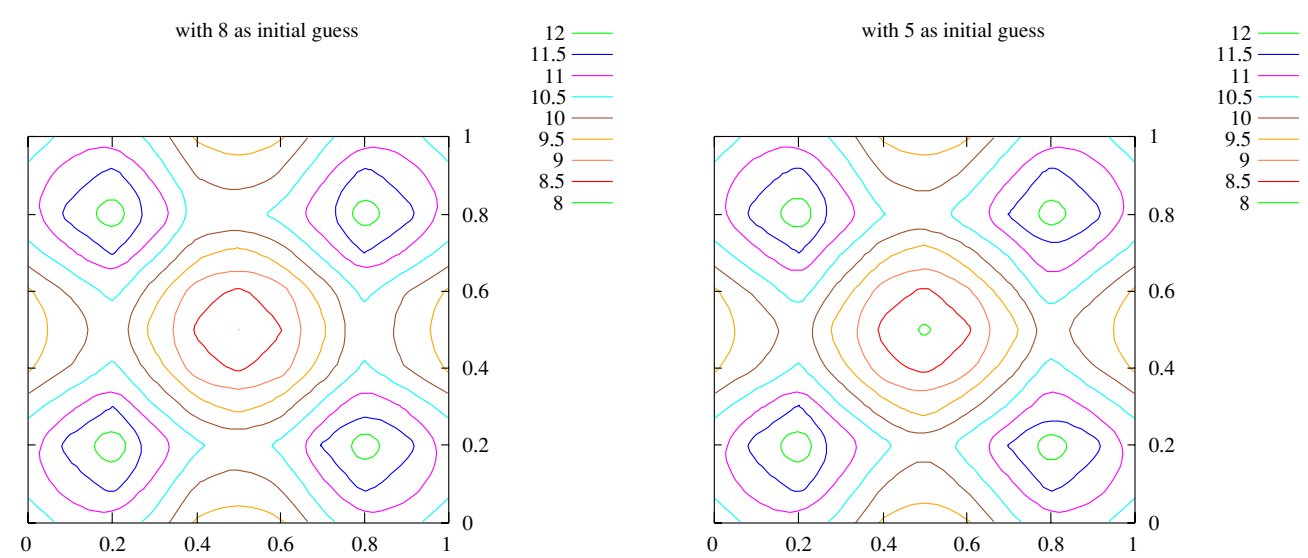

Figure 8. Contour plots of numerical solutions for example 3 with $5 \%$ noise. Left: using 8 as the initial guess. Right: using 5 as the initial guess.

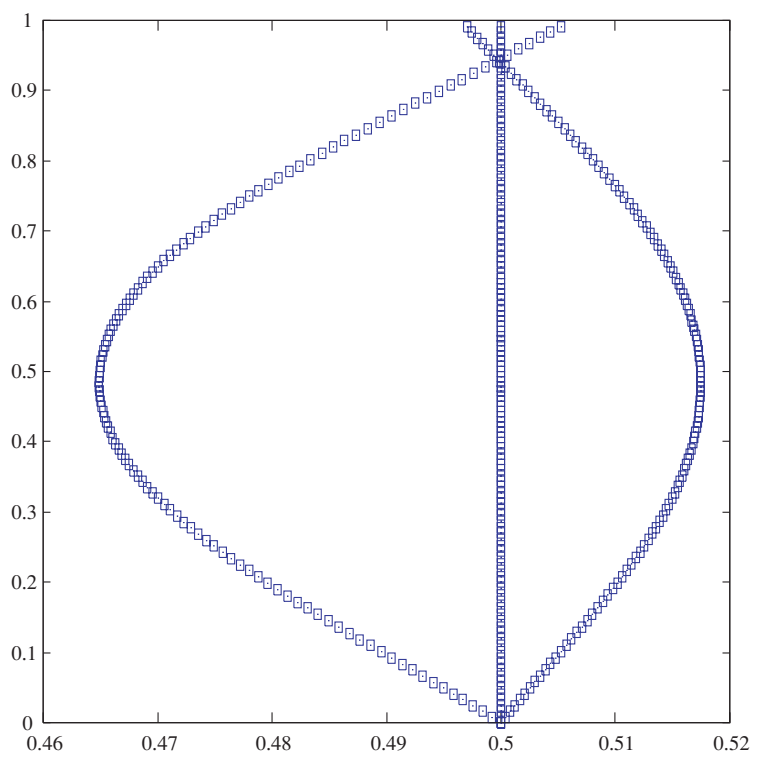

Figure 9. Ray crossing. Multipathing appears in the model.

In figure 10 , the contour plots of the numerical solution and the exact solution are presented.

In figure 11, the numerical and exact solutions at $y=0.5$ and $x=0.5$ are shown respectively.

Figure 12 shows that the rays are crossing, implying that caustics are allowed in our new phase space method. Here, we set the initial location to be $(0.5,0)$ and $(0.7,0)$, respectively, and we use 10 initial directions which are uniformly distributed.

4.1.4. Example 4: the additive sinusoidal model, partially illuminated. To test the robustness of the algorithm, we may use different sets of data to study the convergence behaviour of the 

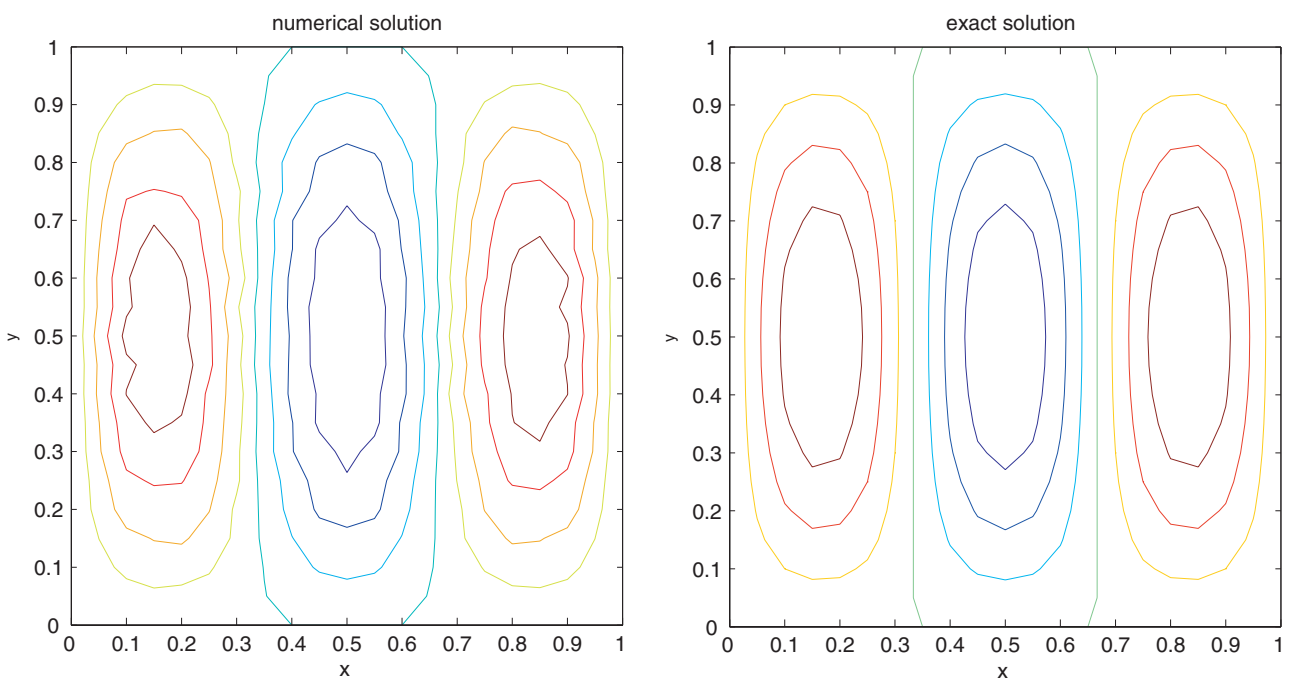

Figure 10. Contour plots for the numerical solution and the exact solution for example 4. Left: numerical. Right: exact.
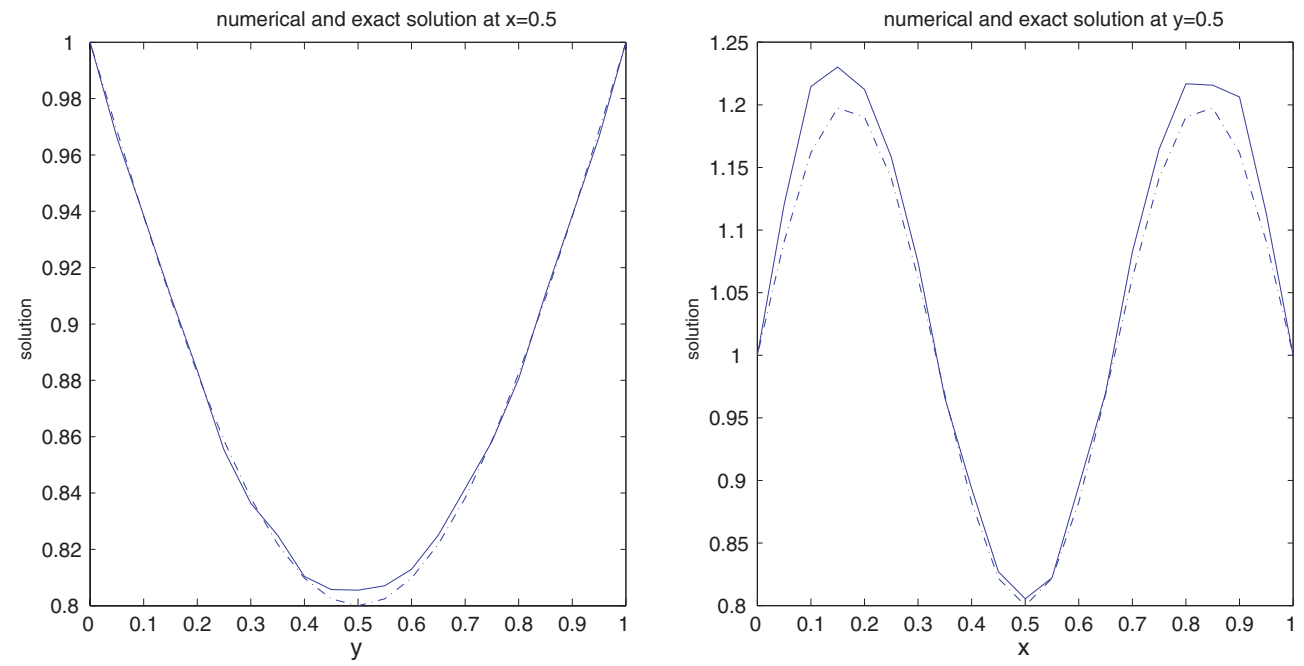

Figure 11. Slices of numerical and exact solutions. Left: $y=0.5$. Right: $x=0.5$. The solid line represents the numerical solution and the dashed line represents the values of the exact solution at the nodal points.

algorithm. We use the following velocity,

$$
c(x, y)=10+\sin (3 \pi x)+\sin (3 \pi y) .
$$

The regularization parameter is $\beta=10^{-4}$. The noise level is $5 \%$. The initial datum is a constant function which is 5 . In this example, we will only use source locations on the edge with $y=0$. That is, there are only 9 initial physical locations. We will demonstrate the effect of the number of measurements by using different numbers of initial directions. More precisely, we consider two cases. The first one is that we will use 100 initial directions for 

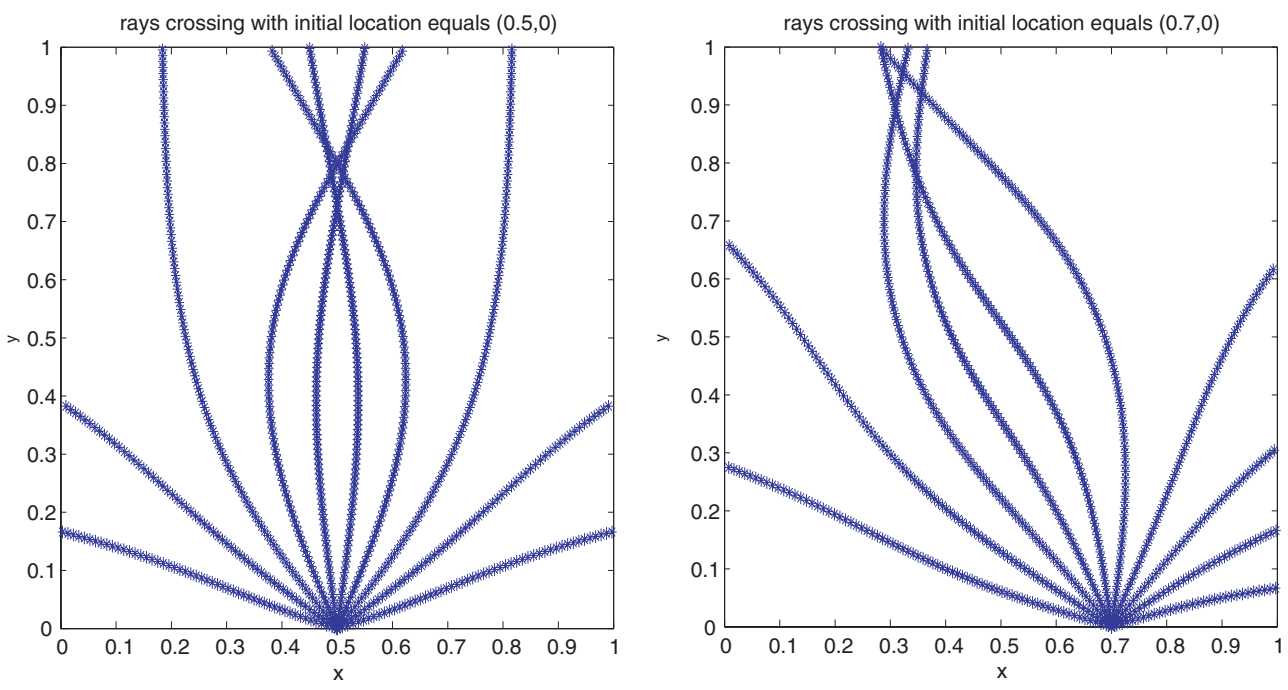

Figure 12. The rays are crossing for example 4. Ten rays emanate from the source location at $(0.5,0)$ and $(0.7,0)$, respectively.
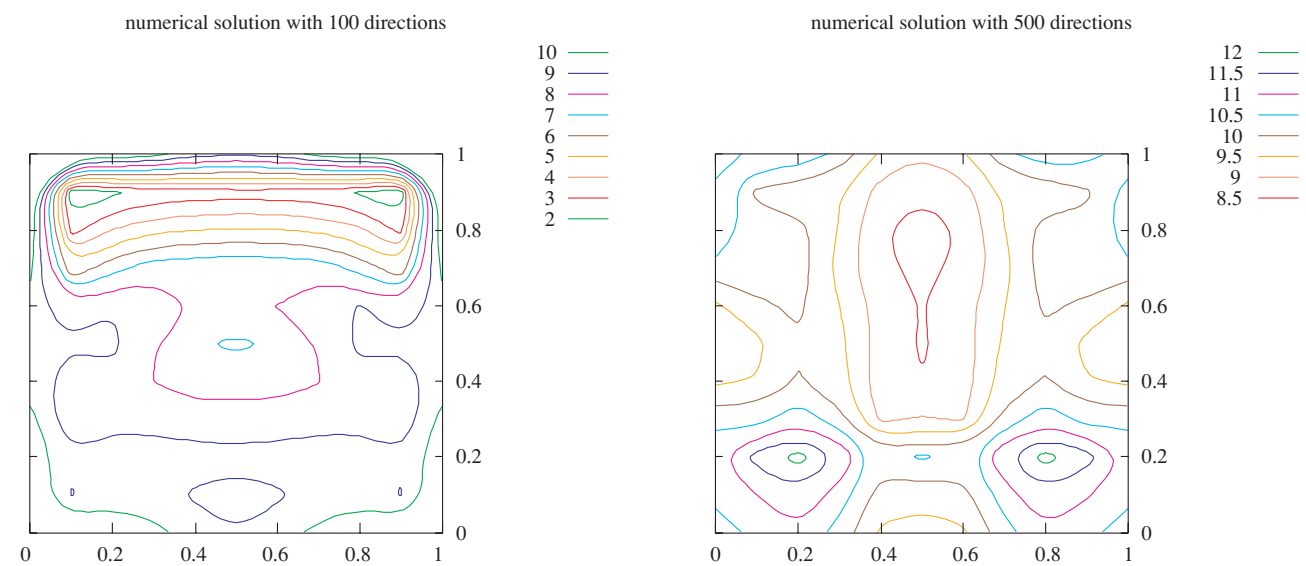

Figure 13. Contour plots of numerical solutions for example 4 with 5\% noise. Left: with 100 directions. Right: with 500 directions.

each of the 9 initial physical locations. The second one is that we will use 500 initial directions for each of the 9 initial physical locations. In figure 13, we show the contour plots of the numerical solutions for both cases. From these results, we see that the numerical solutions are more accurate in regions closer to the $y=0$ boundary. Moreover, the numerical solution with 500 initial directions is more accurate than that with 100 initial directions. The $L^{2}$-norm errors for the two cases are 3.8326 and 0.81848 , respectively.

In figure 14, we plot the slices of the numerical solution and the exact solution at $y=0, y=0.1, y=0.2$ and $y=0.3$. From the figure, we again see that the numerical solution with 500 initial directions is more accurate than that with 100 initial directions. Moreover, we see that the numerical solution is more accurate in regions closer to the boundary where the measurement is made. 

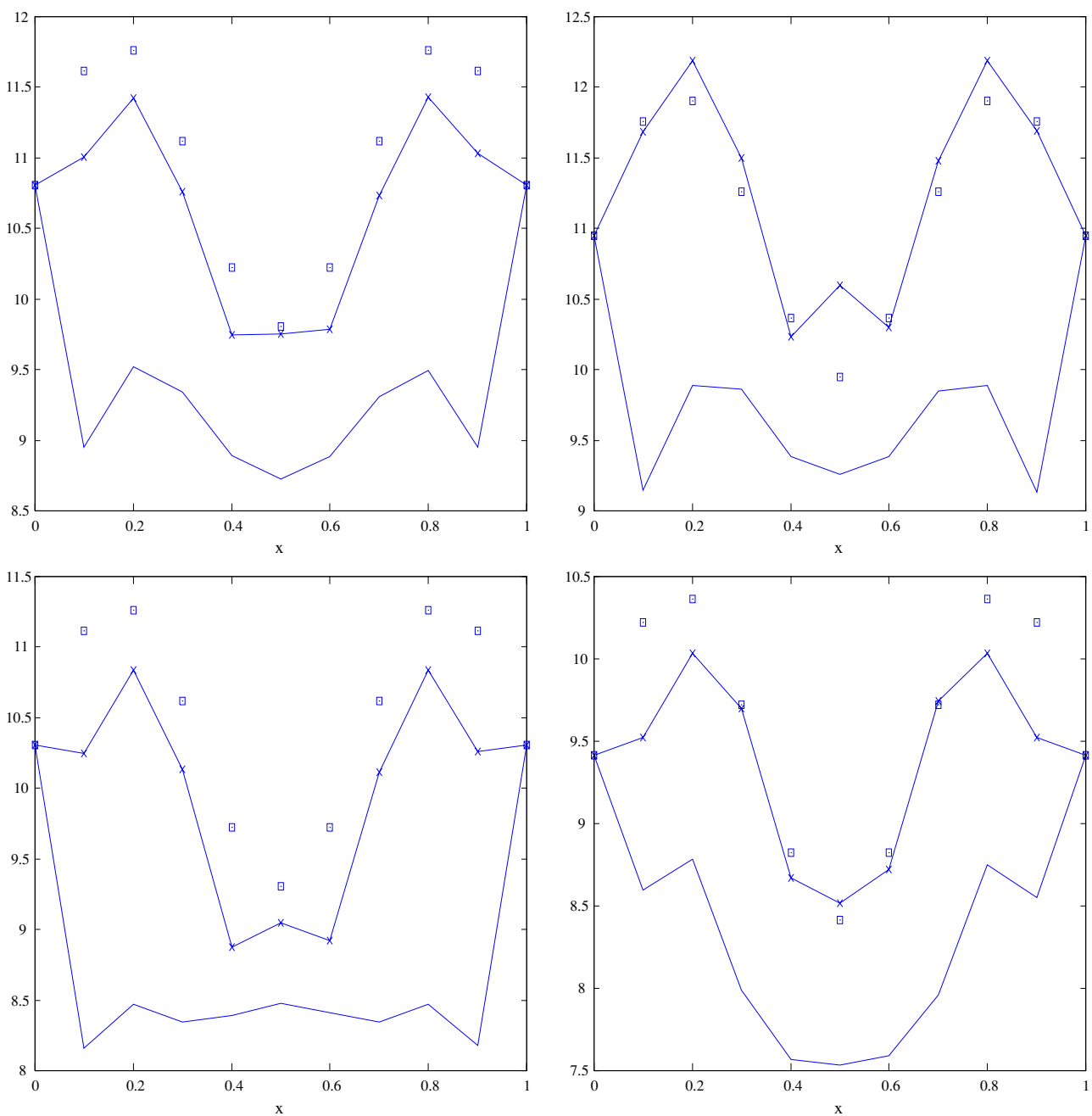

Figure 14. Numerical solution with 100 directions (solid line), numerical solution with 500 directions (solid line with cross), and exact solutions (square) for example 4 with $5 \%$ noise. Top-left: at $y=0.1$. Top-right: at $y=0.2$. Bottom-left: at $y=0.3$. Bottom-right: at $y=0.4$.

Next, we will test the algorithm by using only one initial direction at each source location. The initial direction is chosen such that it is orthogonal to the boundary of the domain. We will study the performance of the algorithm by using 116, 236, 596 and 1196 physical source locations, respectively. These physical source locations are uniformly distributed on the boundary of the domain $\partial \Omega$. In figure 15, the contour plots of the numerical solutions are shown. The $L^{2}$-errors are $1.5429,0.98072,0.63892$ and 0.53205 , respectively. We see that the numerical solution is more accurate when more initial physical locations are used. Also, with only one initial direction at each source point, the numerical method can recover the unknown speed by using a large number of initial physical source locations such that the computational domain is well illuminated.

On the other hand, we remark that the results under partial illumination are not as accurate as those under complete illumination due to the fact that the linearized problem cannot recover 


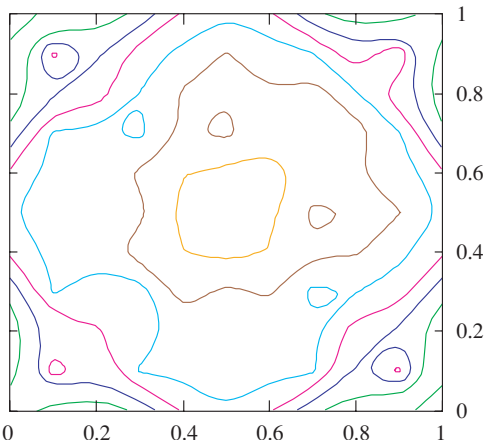

using 596 data

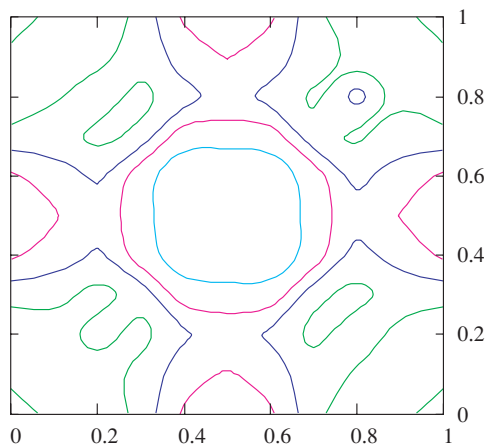

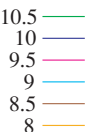

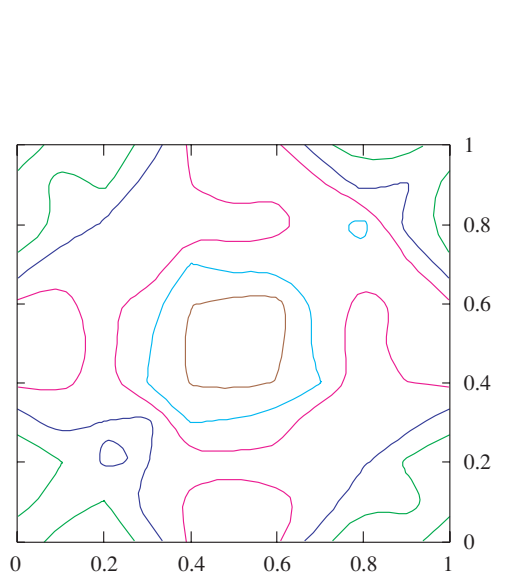

$10.5 \square$
$10 \square$
$9.5 \square$
$9 \square$
8.5

using 1196 data

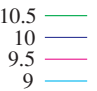

10.5
10
9.5
9

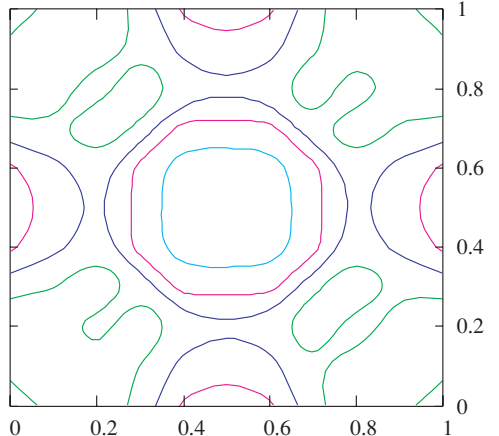

Figure 15. Contour plots of numerical solutions using different numbers of physical source locations and using one initial direction. Top-left: with 116 locations. Top-right: with 236 locations. Bottom-left: with 596 locations. Bottom-right: with 1196 locations.

the wave front set of the coefficient at all directions. This is similar to the well-known phenomenon in $\mathrm{x}$-ray tomography with limited angles of observation.

\subsection{The Marmousi synthetic model}

We test our continuation method on the Marmousi model. The original Marmousi model is defined on a $384 \times 122$ grid. We will take a $122 \times 122$ subset of the original model such that the grid points in the $x$-direction are taken between the 189th grid point and 310th grid point. As a result, the exact solution is defined on a $122 \times 122$ grid, which is shown in the upper-right graph of figure 16 . The measurement is generated by this $122 \times 122$ true speed. We will try to recover the speed on a $21 \times 21$ artificial computational grid.

The initial guess is the constant function 2000 , which is denoted by $g_{0}$. The numerical solution is defined on a $21 \times 21$ grid and the regularization parameter $\beta=10^{-4}$. For 

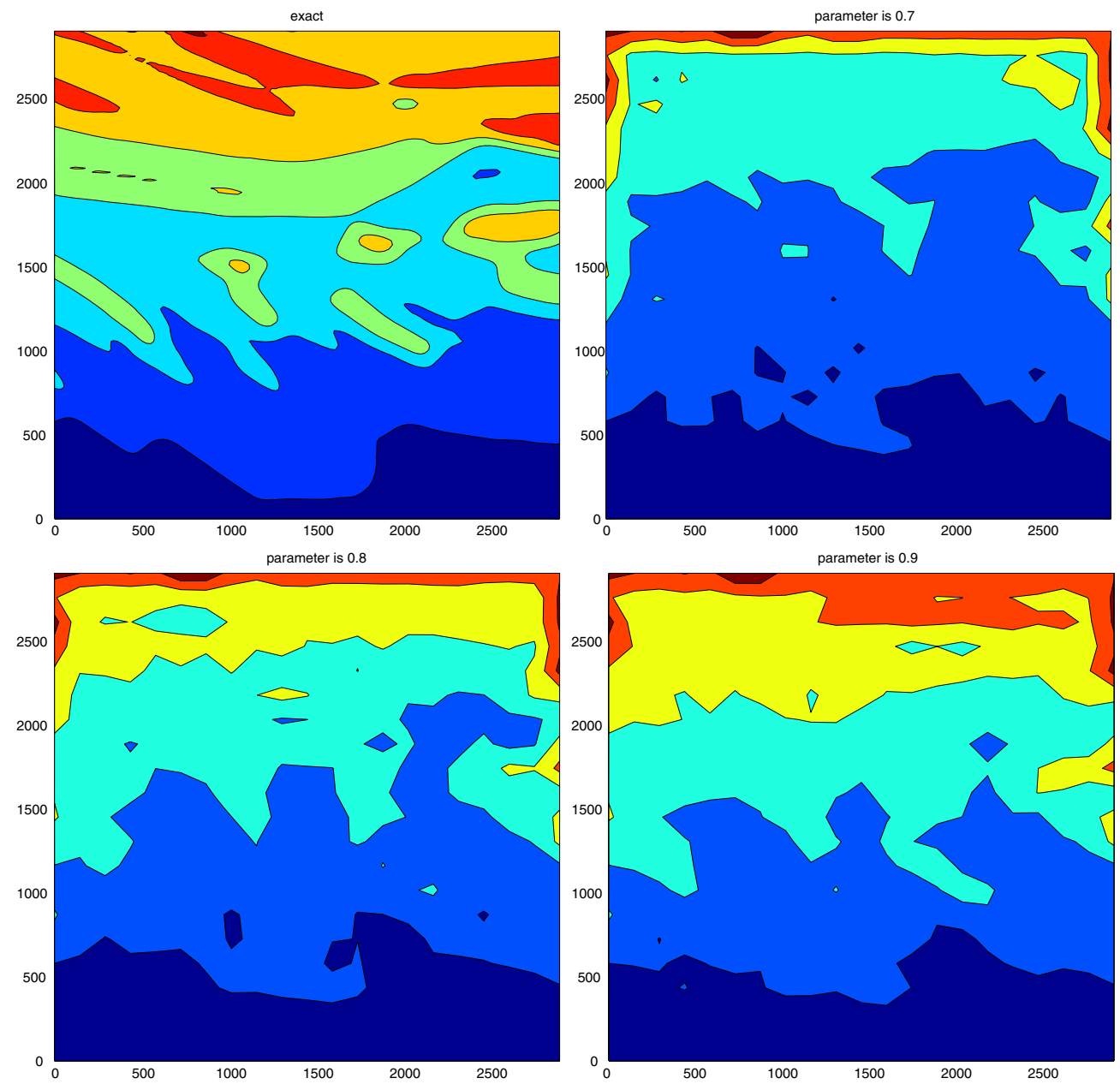

Figure 16. Marmousi example. Top-left: exact. Top-right: with $\alpha=0.7$. Bottom-left: with $\alpha=0.8$. Bottom-right: with $\alpha=0.9$.

$j=1,2, \ldots, m$, we let $\tilde{t}_{j}$ and $\tilde{X}_{j}=X_{g_{0}}\left(\tilde{t}_{j}, X_{j}^{(0)}\right)$ be the solution of the forward problem by using the initial guess as the speed. That is, $\tilde{t}_{j}$ is the travel time when the speed is equal to 2000 while $\tilde{X}_{j}$ is the solution of the forward problem at time $\tilde{t}_{j}$. For each $\alpha=0.1,0.2, \ldots, 1$, we will solve the inverse problem for 5 iterations by using the travel time measurement as $\alpha t_{j}+(1-\alpha) \tilde{t}_{j}$ and the phase measurement as $\alpha X_{\hat{g}}\left(t_{j}, X_{j}^{(0)}\right)+(1-\alpha) \tilde{X}_{j}$. For each $\alpha$, we will use the 5 th iteration from the previous $\alpha$, that is, $\alpha-0.1$, as the initial guess. In figure 16 , we present the numerical solutions for $\alpha=0.7,0.8,0.9$.

As we can see from the figures, the continuation method is able to recover the macro structure of the model reasonably well.

\section{Conclusion}

We have developed a new phase space method for reconstructing the index of refraction of a medium from travel time measurements. The method is based on the so-called Stefanov- 
Uhlmann identity which links two Riemannian metrics in a novel way. Linearizing this identity yields an efficient numerical method for solving the reconstruction problem. In particular, this phase space formulation can deal with multiple arrival times as well as anisotropic media naturally. We have designed numerical algorithms to solve the resulting inverse problem. Numerical examples including isotropic metrics and the Marmousi synthetic model demonstrate the effectiveness of the new method. In a subsequent work we are going to study anisotropic metrics.

\section{Acknowledgments}

Jianliang Qian is supported by NSF \#0542174. The research of Gunther Uhlmann is partially supported by NSF and a Walker Family endowed Professorship. The work of Hongkai Zhao is partially supported by NSF DMS 0513073, ONR Grant \#N00014-02-1-0090, DARPA Grant \#N00014-02-1-0603 and Sloan Foundation Fellowship.

\section{References}

[1] Beylkin G 1983 Stability and uniqueness of the solution of the inverse kinematic problem of seismology in higher dimensions J. Sov. Math. 21 251-4

[2] Bishop T N, Bube K P, Cutler R T, Langan R T, Love P L, Resnick J R, Shuey R T, Spindler D A and Wyld H W 1985 Tomographic determination of velocity and depth in laterally varying media Geophysics 50 903-23

[3] Bube K P and Langan R T 1997 Hybrid $l^{1}-l^{2}$ minimization with applications to tomography Geophysics 62 1183-95

[4] Croke C, Dairbekov N S and Sharafutdinov V A 2000 Local boundary rigidity of a compact Riemannian manifold with curvature bounded above Trans. Am. Math. Soc. 352 3937-56

[5] Dairbekov N S 2006 Integral geometry problem for nontrapping manifolds Inverse Problems 22 431-45

[6] Delprat-Jannaud F and Lailly P 1995 Reflection tomography: how to handle multiple arrivals? J. Geophys. Res. $100703-15$

[7] Engquist B and Runborg O 2003 Computational high frequency wave propagation Acta Numerica 12 181-266

[8] Farra V 1999 Computation of second-order traveltime perturbation by Hamiltonian ray theory Geophys. J. Int. 136 205-17

[9] Gray S 1986 Efficient traveltime calculations for Kirchhoff migration Geophysics $\mathbf{5 1} 1685-8$

[10] Harlan W and Burridge R 1983 A tomographic velocity inversion for unstacked data Stanford Exploration Project SEP37-01

[11] Isakov V 1993 Uniqueness and stability in multidimensional inverse problems Inverse Problems $9579-621$

[12] Isakov V 1998 Inverse Problems for Partial Differential Equations (New York: Springer)

[13] Isakov V and Sun Z 1992 Stability estimates for hyperbolic inverse problems with local boundary data Inverse Problems 8 193-206

[14] Leung S and Qian J 2005 Transmission traveltime tomography based on paraxial Liouville equations and level set formulations Preprint

[15] Leung S and Qian J 2006 An adjoint state method for three-dimensional transmission traveltime tomography using first-arrivals Commun. Math. Sci. 4 249-66

[16] Liu Z and Bleistein N 1995 Migration velocity analysis: theory and an iterative algorithm Geophysics $60142-53$

[17] Locker J and Prenter P M 1980 Regularization with differential operators: I. General theory J. Math. Anal. Appl. 74 504-29

[18] Locker J and Prenter P M 1980 Regularization with differential operators: II. Weak least squares finite element solutions to first kind integral equations SIAM J. Numer. Anal. 17 247-67

[19] May B T and Covey J D 1981 An inverse ray method for computing geologic structures from seismic reflectionszero-offset case Geophysics 46 268-87

[20] Michel R 1981 Sur la rigidite imposee par la longueur des geodesiques (On the rigidity imposed by the length of geodesics) Invent. Math. 65 71-83 (in French)

[21] Mukhometov R G 1977 The reconstruction problem of a two-dimensional Riemannian metric, and integral geometry Dokl. Akad. Nauk SSSR 232 32-5 (in Russian)

[22] Mukhometov R G 1982 On a problem of reconstructing Riemannian metrics Siberian Math. J. 22 420-33 
[23] Pestov L and Uhlmann G 2005 Two dimensional compact simple Riemannian manifolds are boundary distance rigid Ann. Math. 161 1093-110

[24] Romanov V G 1987 Inverse Problems of Mathematical Physics (Utrecht: VNU Science Press)

[25] Sei A and Symes W W 1994 Gradient calculation of the traveltime cost function without ray tracing 65th Ann. Int. Mtg, Soc. Expl. Geophys., Expanded Abstracts (Tulsa, OK: Soc. Expl. Geophys.) pp 1351-4

[26] Sei A and Symes W W 1995 Convergent finite-difference traveltime gradient for tomography 66th Ann. Int. Mtg, Soc. Expl. Geophys., Expanded Abstracts (Tulsa, OK: Soc. Expl. Geophys.) pp 1258-61

[27] Sharafutdinov V A 1994 Integral Geometry of Tensor Fields (Utrecht: VSP)

[28] Stefanov P and Uhlmann G 1998 Rigidity for metrics with the same lengths of geodesics Math. Res. Lett. 5 83-96

[29] Stefanov P and Uhlmann G 2005 Boundary rigidity and stability for generic simple metrics J. Am. Math. Soc. 18 975-1003

[30] Stefanov P and Uhlmann G 2005 Recent progress on the boundary rigidity problem Electron. Res. Announc. Am. Math. Soc. 11 64-70

[31] Stefanov P and Uhlmann G 2006 Boundary and lens rigidity, tensor tomography and analytic microlocal analysis Proc. Conf. in Honour of Prof. T. Kawai (Tokyo: Springer) at press

[32] Stefanov P and Uhlmann G 2006 Integral geometry of tensor fields on a class of non-simple Riemannian manifolds, submitted

[33] Sword C H 1987 Tomographic determination of interval velocities from reflection seismic data: the method of controlled directional reception PhD Thesis Stanford University, Stanford, CA 94305

[34] Taylor M E 1996 Partial Differential Equations: Basic Theory (New York: Springer)

[35] Wang J-N 1999 Stability for the reconstruction of a Riemannian metric by boundary measurements Inverse Problems 15 1177-92

[36] Washbourne J K, Rector J W and Bube K P 2002 Crosswell traveltime tomography in three dimensions Geophysics 67 853-71 